GANIT J. Bangladesh Math. Soc. (ISSN 1606-3694) 38 (2018) 89-104

DOI: https://doi.org/10.3329/ganit.v38i0.39789

\title{
A BYPASSING TECHNIQUE FOR THE REMEDY OF PORTAL HYPERTENSION THROUGH EXTRA HEPATIC PORTAL VEIN OBSTRUCTION BY CFD ANALYSIS
}

\author{
Mst. Khorseda Atkar* And Md. Tajul Islam \\ Department of Mathematics, Begum Rokeya University, Rangpur-5400, Bangladesh \\ *Corresponding author: keyecard@gmail.com
}

Received: 26-05-2018

Accepted: 17-11-2018

\begin{abstract}
Extra-hepatic portal vein obstruction (EHPVO) is the blockage to the flow of blood in the portal vein before reaches to the liver. EHPVO is the common cause of portal hypertension in children in the most Asian countries. Examination reveals that the presence of block in the main portal vein may be responsible for the shrinkage of vein with manifold pernicious complication. The "shunt" policy is a fruitful source of restoration of the hepatic portal flow. This study shows that a new approach of bypassing (or shunting) to the blocked (thrombosed) region of the portal vein is a significant way of reducing portal hypertension and restoration of blood circulation. We studied EHPVO case through computational fluid dynamics (CFD) analysis by considering partial block formation and side to side shunt scheme inside the main portal vein. The constitutive equation for non-Newtonian fluidand energy equation are solved by control volume technique. Our study reveals that the shunting technique is strongly effective for the reconstitution of portal venous flow to the liver with lower tissue stress and rapid regression of clinical signs of portal hypertension. This new technique may potentially applicable for medication of EHPVO when shunting procedures are indicated.
\end{abstract}

Key words: Portal hypertension, Extrahepatic portal vein obstruction, computational fluid dynamics, Shunt, lower tissue stress.

\section{Introduction}

The portal vein (PV) is formed by the confluence of the splenic vein and the superior mesenteric vein behind the neck of the pancreas. Portal vein is responsible for around two thirds of the blood flow that rich in oxygen, nutrients growth factors, hormones, among other elements. In the hilum of the liver, the portal vein divides into the left and right portal vein branches which supply blood to the left and right sides of the liver respectively.

The obstruction of blood flow within the portal circulation occur 'Portal Hypertension'. This obstruction can be intrahepatic, pre-hepatic or post-hepatic. Portal hypertension ( $\mathrm{PH})$ is used to describe elevated pressures in the portal venous system. The elevated pressure is characterized by a gradient of greater than $5 \mathrm{~mm} \mathrm{Hg}$ between the portal venous and central venous pressures. Portal 
hypertension, also caused by intrinsic liver disease or structural changes, may increase portal venous flow or hepatic resistance. Sherlock [21] classified, an older classification, PH into two main groups - presinusoidal and intrahepatic. The presinusoidal is further divided into extrahepatic, in which the obstruction is in the main portal vein.

Portal vein thrombosis (PVT) is an important cause of portal hypertension. PVT refers to thrombosis that develops in the trunk of the portal vein or including its right and left intrahepatic branches and may even extend to the splenic or superior mesenteric veins or towards the liver involving intrahepatic portal branches. PVT occurs either in association with cirrhosis or malignancy of liver or may occur without an associated liver disease.

Balfour and Stewart [1] described the first case of PVT in 1868 in a patient with ascites, splenomegaly and variceal dilation. Non-cirrhotic prehepatic portal hypertension is a consequence of portal vein thrombosis all over the world.

Orllof et al [2] determined the site of blood flow obstruction in 200 children and young adults. The authors observed that $67 \%$ of patients showed only portal vein obstruction, whereas $28 \%$ showed portal and splenic vein involvement, and 5\% had portal vein and superior mesenteric thrombosis. Portal vein obstruction can occur at any site of the portal system.

When portal hypertension occurs in the absence of cirrhosis, Extra Hepatic Portal Venous Obstruction (EHPVO) is most often indicated etiologically. Patients with EHPVO are generally young and belong mostly to Asian countries. EHPVO is an important cause of PH among children. It is characterized mainly by PVT and is detected with upper gastrointestinal bleeding caused by esophageal varices [3]. The EHPVO has been showed a significant enlargement of the spleen (16$17 \mathrm{~cm}$ ) with signs of portal hypertension. The liver appeared to be slightly enlarged with a normal echo-pattern and regular borders [4].

When portal hypertension occurs, the most common solution to restoring the normal blood flow is to use shunts. A shunt is a graft, which takes the blood from one part of a blood vessel to another or from one blood vessel to another to bypass problem areas in the vessel.

There are different types of shunts such as side-to-side, end-to side and transjungualar intrahepatic portosystemic shunt (TIPS) have been used for the restoration of portal flow. However, in most cases these are used only as a temporary assist device and have to be replaced after some time due to thrombosis, graft failure and platelet adhesion on the graft. The shunts are usually used only as a short-term solution to assist medication. A more permanent solution to these problems is to create a bypass or shunt which has minimally thrombogenic properties, from a minimally platelet adhesive material with a high degree of durability.

The material from which a shunt or bypass is made generally can be classified as: synthetic (manmade), autograft (from another vessel of the same patient), homograft (from another human donor) or animal modified vessel (treated to be non-antigenic). Nowadays, it is regular practice to create a 
bypass and shunt from another vessel of the patient. There are many benefits to this method compared to the synthetic grafts or other homografts including high biocompatibility, low risk of rejection and lower tissue stress.

Children with extrahepatic portal obstruction either from thrombosis of the portal vein or extrinsic portal vein compression who do not respond to conservative measures or in whom clinically significant hypersplenism develops have been shown to benefit from surgical decompression of the variceal bed by portosystemic shunting. Surgical options recommended for portal hypertension in children have included mesocaval shunts [5-6], gastric devascularization procedures [7-8] and the distal splenorenal shunt [9-10].

Other reports show limited success for portal hypertension decompression with TIPS due to early thrombosis $(12 \%)$, stenosis $(41 \%)$ and high re-bleeding rate [19]. In patients with good liver function elective operation might be more beneficial than TIPS [19], and the latter is more beneficial for patients with poor liver function, active bleeding or liver transplant candidates.

In patients with refractory ascites (study with 65 patients [20]) TIPS are associated with unpredictable and high rates of mortality and morbidity.

Goyet et al [11] has used a venous autograft between the superior mesenteric vein and the distal (umbilical) portion of the left portal vein to achieve hepatic portal revascularisation and decompression of extrahepatic portal hypertension in children with cavernoma and obstruction caused by idiopathic portal vein thrombosis.

Computational Fluid Dynamics (CFD) has shown a great potential in computational biology and biomechanics lately due to the limitations of traditional measurement methods. Since CFD uses numerical methods and algorithms to solve and analyze problems that involve fluid flows so its applicability can be exploited for solving blood flow problems. This method can render relatively accurate results of the flow system.

In the study of portal system hemodynamic, Botar et al [12] implemented the Reynolds Stress model to describe hemodynamic in portal vein system. He used actual patient imaging of the portal vein in CFD modeling to obtain hemodynamic quantities such as velocities and wall shear stress. A very good correlation between simulation results and clinical data has been obtained by this study.

Petkova et al [13] examined blood flow in idealized model of the portal vein with and without thrombosis using CFD with both a Newtonian and a Non-Newtonian blood viscosity model. The main focus of this study is that to evaluate the impact of thrombosis on the flow behavior.

Aktar et al [14] carried out a quantitative analysis of inconsistence blood flow through portal vein under different sizes of block using computational fluid dynamics. This study showed that the presence of block in the main portal vein may be responsible for the shrinkage of vein with many complication of liver diseases. 
The aim of this research is to emphasize the importance of repairing acute venous blockage to overcome portal hypertension and provide a long-term solution via an autograft blood vessel and/or better understanding of blood flow characteristics using computer modelling.

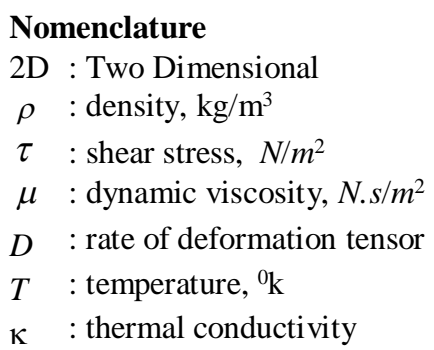

\section{Model Development}

\subsection{Problem Statement}

As seen in previous reports, the thrombosis is often limited to the portal trunk and does not extend into the intrahepatic branches [15-16]. Here we consider Extra Hepatic Portal Vein Obstruction (EHPVO) by forming partial block in the main portal vein (without involving intrahepatic obstruction). We also consider shunting procedure for directly bypassing the thomobosed region of the portal vein.This technique is potentially applicable for the decompression of the portal hypertension, as a complication of the Extra Hepatic Portal Vein Obstruction. The thrombosed region is bypassed by interposing a vein like channel between the portal vein and the distal portion of the left branch of the portal vein. We have used a simple 2D geometry of portal vein as shown in fig.1 and fig.2.

Fig.1 exhibits a portal vein with partial blocked in the main portal vein which is actually a shrink vein due to the formation of the thrombosis. A directed bypassing of the thomobosed region is revealed by fig. 2 .

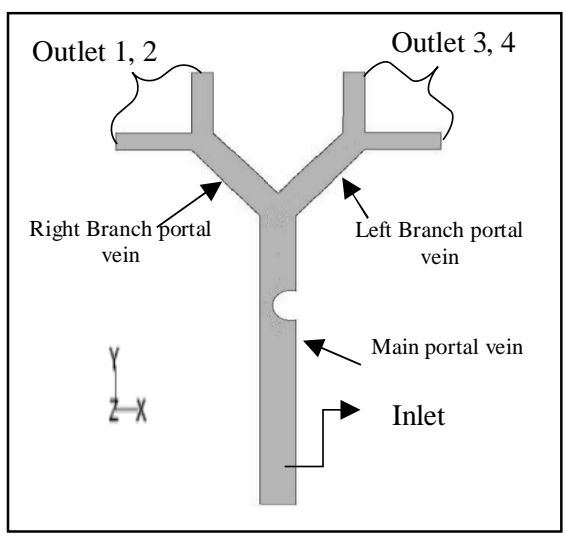

Fig.1: $85 \%$ partially blocked portal vein

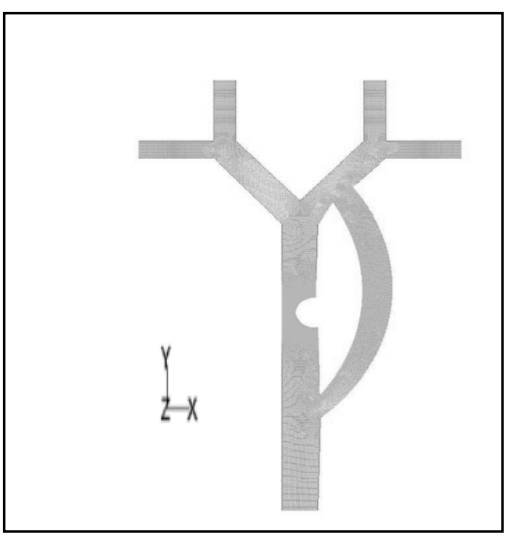

Fig.2: A portal vein with partial shunt 
Table 1 shows the dimensions of the geometry used in this model.

Table 1: Model dimensions

\begin{tabular}{|l|l|}
\hline Description & Dimensions $(\mathrm{mm})$ \\
\hline Inlet diameter & $10 \mathrm{~mm}$ \\
\hline First branching diameter & $8 \mathrm{~mm}$ \\
\hline Outlet diameter & $6 \mathrm{~mm}$ \\
\hline Diameter of the shunt & $10 \mathrm{~mm}$ \\
\hline Total height & $150 \mathrm{~mm}$ \\
\hline
\end{tabular}

The model geometry was constructed by using workbench a component of the ANSYS package. The meshing scheme is composed of elements and type. The elements selected were quadrilateral defines the shape of the element used to mesh and the type parameter defines the meshing algorithm which in our case was pave. After the geometry is successfully meshed, the zone types are specified. A mesh file is exported for use in Fluent 17.2.

\subsection{Governing Equations}

The mass and linear momentum conservation equations which govern the flow of any fluid are respectively

$\frac{\partial \rho}{\partial}+\nabla \cdot(\rho \vec{V})=0$

$\rho \frac{\partial \vec{V}}{\partial t}+\rho \vec{V} \cdot \nabla \vec{V}=\rho \vec{g}-\nabla P+\nabla \cdot \vec{\tau}$

Where, $\rho$ denotes the density of the fluid, $\vec{V}$ is the velocity vector, $\mathrm{P}$ is the pressure, $\vec{\tau}$ is deviatoric stress, $\vec{g}$ is the gravitational body force and $t$ is time. It is assumed that $\vec{\tau}$ is a symmetrical tensor, thus ensuring that angular momentum is conserved.

Determination of the flow of any fluid requires solution of these conservation equations together with constitutive equations for the fluid. It is in the constitutive equations that the nature of a given fluid is manifested.

For a Newtonian fluid $\vec{\tau}$ is given by:

$\vec{\tau}=\mu D$

Where $D$ denotes the rate of deformation tensor defined by

$D=\nabla \vec{V}+(\nabla \vec{V})^{T}$

and $\mu$ denotes the viscosity of the fluid which is independent of $D$ (though it may, of course, depend on temperature $\mathrm{T}$ and perhaps on pressure $\mathrm{p}$ ). A non-Newtonian fluid is one for which Eq. (3) does not hold or one for which Eq. (3) holds but only with $\mu$ dependent on $D$. 
Since our model is based on shear flow, the relevant rheological or flow property is viscosity $\mu$ and $\vec{\tau}$ is given by (3). A fluid for which (3) holds but $\mu$ depends on $D$ is called a generalized Newtonian fluid.

If $\mu$ depends on $D$, then $\mu$ must be a function of the three scalar invariants $\mathrm{I}_{\mathrm{D}}, \mathrm{II}_{\mathrm{D}}$ and $\mathrm{III}_{\mathrm{D}}$ of $D$ which are given by:

$$
\begin{aligned}
& I_{D}=\operatorname{tr}(D) \\
& \mathrm{II}_{D}=\frac{1}{2}\left[\operatorname{tr}\left(D^{2}\right)-(\operatorname{tr}(D))^{2}\right] \\
& \mathrm{III}_{D}=\operatorname{det}(D)
\end{aligned}
$$

Where $\operatorname{tr}($ ) and det ( ) denote trace and determinant, respectively. For an incompressible fluid, the incompressibility conditions ensure that

$$
\mathrm{I}_{D}=\operatorname{tr}(D)=0
$$

A shear flow is one for which:

$$
\mathrm{III}_{D}=\operatorname{det}(D)=0
$$

Thus $\mathrm{II}_{\mathrm{D}}$ is the only nonzero invariant for a shear flow of an incompressible fluid. Hence the shearrate, denoted by $\dot{\gamma}$, is defined by:

$$
\dot{\gamma}=\sqrt{I I_{D}}
$$

Hence the constitutive equation for the stress $\vec{\tau}$ of an incompressible non- Newtonian fluid is:

$$
\vec{\tau}=\eta(\dot{\gamma}) D
$$

Where $\eta$ is non-Newtonian viscosity or apparent viscosity of fluid.

In order to take into account the non-Newtonian viscosity of blood in the numerical simulation of hemodynamic flow, we define the relationship between viscosities and shear rate by power-law model as follows:

$$
\eta(\dot{\gamma})=K \dot{\gamma}^{n-1}
$$

Where $n$ is called the power-law index and Kis called the consistency factor.

Thus, the stress tensor for a power-law fluid is defined as:

$$
\vec{\tau}=K\left|I I_{D}\right|^{\frac{n-1}{2}} D
$$

Where $\mathrm{II}_{D}=\frac{1}{2} \operatorname{tr}\left(D^{2}\right)$

Using equation (11) in equation (2) then it becomes the following form

$$
\rho \frac{\partial V}{\partial t}+\rho \vec{V} \cdot \nabla \vec{V}=\rho \vec{g}-\nabla P+\nabla \cdot K\left|I I_{D}\right|^{n-1} D
$$


In our study, the 2D blood flow is assumed to be steady and laminar. The three basic laws of conservation of mass, momentum and energy are solved for incompressible flows of nonNewtonian fluid.

The mass conservation and the constitutive equation of non-Newtonian fluid can be written as follows

$$
\begin{aligned}
& \Delta .(\vec{V})=0 \\
& \rho(\vec{V} . \nabla) \vec{V}=\rho \vec{g}-\nabla P+\nabla \cdot K(\nabla \vec{V})^{n}
\end{aligned}
$$

When, $n$ is equal to one the Navier-stokes constitutive equation is recovered. When $n$ is less one than, the constitutive equation is shear thinning while $\eta$ is greater one than, the behavior is shear thickening.

Once the local shear rate is calculated, the non-Newtonian viscosity $\eta$ can be calculated by using the following formula (FLUENT 6.0 Manual, Chapter 7.3.5)

$$
\eta=K \dot{\gamma}^{n-1} e^{\frac{T_{0}}{T}}
$$

Where $\mathrm{K}$ the power law consistency factor, $n$ is the flow behavior index, $T_{0}$ is the reference temperature.

Fluent allows upper and lower limits to be placed on the power law function, yielding the following equation:

$$
\eta_{\min }<\eta=K \dot{\gamma}^{n-1} e^{\frac{T_{0}}{T}}<\eta_{\max }
$$

Where, $K, \mathrm{n}, \mathrm{T}_{0}, \eta_{\min }$ and $\eta_{\max }$ are input parameters, $\eta_{\min }$ and $\eta_{\max }$ are respectively the lower and upper limits of non-Newtonian viscosity used in power law. If the viscosity computed from the power law is less than $\eta_{\min }$, the value of $\eta_{\min }$ will be used instead. Similarly, if the computed viscosity is greater than $\eta_{\max }$, the value of $\eta_{\max }$ will be used. Table 2 shows how viscosity is limited by $\eta_{\min }$ and $\eta_{\max }$ at low and high shear rates in this model.

The Energy equation is

$$
\rho \operatorname{div}(i \vec{V})=-P \operatorname{div} \vec{V}+\operatorname{div}(\kappa \operatorname{grad} T)
$$

Where $i$ is the specific internal energy, $\vec{V}$ is the velocity field, $\kappa$ is the thermal conductivity and $\mathrm{T}$ is the temperature of fluid. In case of incompressible flow there is no need to establish linkage between the energy equation and the equations of continuity and momentum.

\subsection{Basic Assumption}

The portal vein or hepatic portal vein is a blood vessel that carries blood from the gastrointestinal tract and spleen to the liver. The blood from the portal vein supplies $80 \%$ of the liver's blood and contains nutrients that were absorbed from the digestive tract. As the heart pulsates blood at a 
certain frequency, the blood that flows from that organ has a pulsatile behavior. However as the blood flows to the rest of the body and the blood vessels get thinner, the blood flow assumes a steady state behavior. In mathematical point of view two parameters are used to know whether the flow is closer to steady or pulsatile behavior. Those parameters are Reynolds number $(\mathrm{Re})$ and the Womersely number $(\alpha)$. The following equations are used to determine the value of $\operatorname{Re}$ and $\alpha$

$$
\begin{aligned}
\operatorname{Re} & =\frac{\rho \vec{V} D}{\mu} \\
\text { and } \quad \alpha & =\frac{D}{2} \sqrt{\frac{\rho \omega}{\mu}}
\end{aligned}
$$

Where, $\rho$ is the blood density, $\vec{V}$ is the mean velocity, $\mathrm{D}$ is the diameter of the blood vessel, $\omega$ is the angular frequency, $\omega=2 \pi \times 0.5 \mathrm{rad} / \mathrm{s}$ and $\mu$ is the blood viscosity. In our model, the Womersely number is $\alpha=5.26$ according to equation (19). Since $\alpha=10$, flow can be assumed steady. Reynolds number is $\operatorname{Re}=708$ according to equation (18) so the flow is laminar.

Blood viscosity can be defined as the quantity that describes the blood's resistance to flow, which is being deformed by either shear stress or tensile stress. For different parts of the circulatory system, different behaviors of blood viscosity seem to happen. In healthy straight large blood vessels, where the blood flow is in high strain rate blood shows Newtonian behavior, however, in small blood vessels, the strain rate is low and the blood viscosity shows a shear-thinning behavior.

\subsection{Boundary Conditions}

In every CFD model, apart from the geometry definition and the consequent mesh, there is the need of prescribing boundary conditions. Blood flows by entering and exiting blood vessels, which have walls that do not allow blood to pass through. Therefore it is clear that for the inlet and outlet as well as for the walls there must be boundary conditions. For the inlet a mass-flow rate is used. As for the outlets an outflow condition is used. Rigid wall conditions were assumed according to literature [12] and neglecting elastic property [17].

For our study, we consider inlet as mass-flow rate under portal hypertension condition. The operating pressure is 2737.1 Pascal. Since the left part of liver is approximately two times smaller than the right part and therefore has a blood flow twice as high [2]. All outlets were classified as outflow in which the flow spit was taken with flow rate weighting 0.70 for each outlet 1 and 2 in the right branch and 0.50 for each of outlet 3 and 4 in left branch. Portal vein carries rich blood of nutrients which has large amount of water (approximately 70\%). For this reason we consider alikeblood as working fluid whose physical properties (specific heat and thermal conductivity) are similar to water with density $\rho=1060 \mathrm{~kg} / \mathrm{m}^{3}$. As said previously, blood shows a shear-thinning behavior and is often modeled as a Non-Newtonian fluid. This behavior is dependent on the strain rate of fluid. However, a diseased portal vein with decreased blood flow will show a lower strain rate in the range of $1-200 \mathrm{~s}^{-1}$ where the shear-thinning will be important [18]. 
The flow properties of blood used in this study are given in the following table.

Table 2: Flow properties

\begin{tabular}{|l|l|}
\hline Power law index $(\mathrm{n})$ & 0.4851 \\
\hline Consistency index $\kappa\left(\mathrm{kg}-\mathrm{s}^{\mathrm{n}-2} / \mathrm{m}\right)$ & 0.2073 \\
\hline Reference temperature $\left({ }^{0} \mathrm{~K}\right)$ & 310 \\
\hline Minimum viscosity limit $\eta_{\max }(\mathrm{kg} / \mathrm{m}-\mathrm{s})$ & 0.00125 \\
\hline Maximum viscosity limit $\eta_{\min }(\mathrm{kg} / \mathrm{m}-\mathrm{s})$ & 0.003 \\
\hline
\end{tabular}

\subsection{Numerical Method}

The pressure based solver was chosen due to the modeling of low-speed incompressible flow. FLUENT solves the governing equations for mass and momentum in order to obtain thevelocity field. To do this a control volume based technique is used. The domain is divided into discrete control volumes based on the computational grid. The governing equations are integrated over the individual control volumes to construct algebraic equations. The discretized equations are linearized and solved to yield values for unknown variables. The formulation for the solver was implicit. The velocity formulation was absolute and porous formulation was superficial velocity. The Green-Gauss Cell based gradient option was used. For the pressure-velocity coupling the SIMPLE (Semi-Implicit Method for Pressure-Linked Equations) algorithm was selected which uses the relationship between velocity and pressure correction to enforce mass conservation and obtain the pressure field. The computations are considered to be converged when the residues for continuity, momentum and energy are less than $10^{-6}$.

\subsection{Grid dependency Test}

To evaluate the grid size effect, grid dependency tests are carried out. Three different sizes of grid $80 \times 12000,90 \times 15000$ and $100 \times 18000$ are tested for the typical domain as shown in fig. 1 for incompressible flow with non-slip boundary conditions and the results are listed in Table 3 . The table shows that the difference of average velocity at outlet for grid sizes $80 \times 12000,90 \times 15000$ and $100 \times 18000$ respectively.

For convenience, we used grid size $100 \times 18000$ or its multiple according to the length of the dimensions of the domain.

Table 3: Grid dependency test of the average velocity under three sizes of grids

\begin{tabular}{|l|l|}
\hline Grid size & Average velocity \\
\hline $80 \times 12000$ & 0.992931 \\
\hline $90 \times 15000$ & 0.9923801 \\
\hline $100 \times 18000$ & 0.9987421 \\
\hline
\end{tabular}




\subsection{Validity and Verification}

To validate our model we simulated blood flow through a normal portal vein (without block) with the boundary conditions as was done by Petkova [13]. We compared velocity distribution with that of Petkova at $\mathrm{y}=20 \mathrm{~mm}$. As displayed in Fig.3, the results mostly agreed with Petkova model results except for minor differences which are due to 3D geometry used by Petkova. We would proceed with the assumption that our model is a valid model.

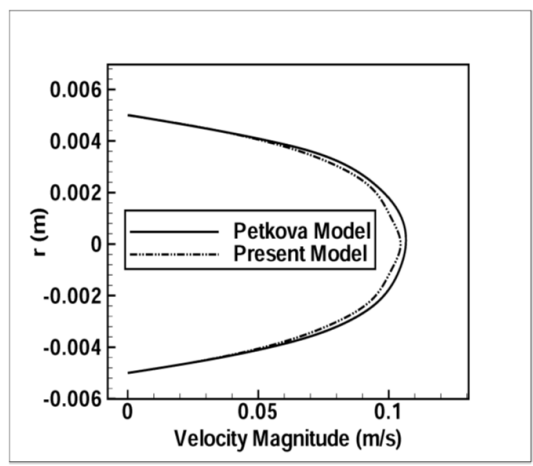

\section{Results}

Fig.3: The velocity distribution at $\mathrm{y}=20 \mathrm{~mm}$

The simulation results of blood flow in the partial blocked (or thomobosed) portal vein as well as portal vein with side to side shunt (a bypass of the thomobosed region) have been rendered in this section. The comparison of pressure distribution of blood flow through the partial blocked portal vein $(\mathrm{PV})$, along the streamwise direction whichare at $\mathrm{y}=20 \mathrm{~mm}$ and $\mathrm{y}=80 \mathrm{~mm}$ respectively, have shown in fig.4.

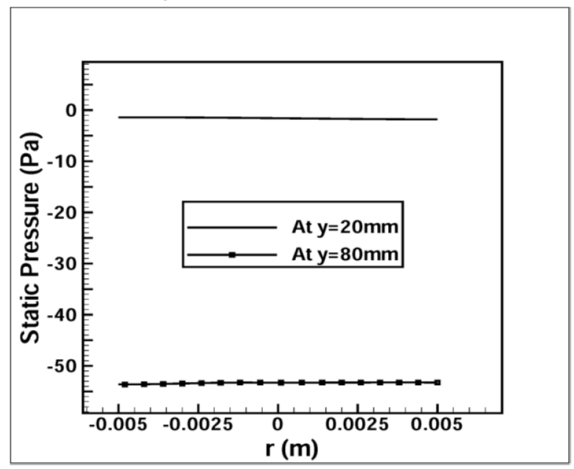

Fig.4: Pressure distributions for partial blocked portal vein case

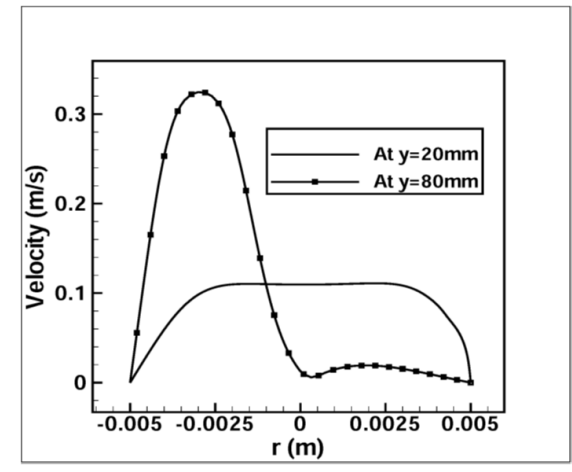

Fig.5: Velocity distributionsfor partial blocked portal vein case

It is clearly shown in fig. 4 that the pressure taken at $y=20 \mathrm{~mm}$ is much higher than the pressure at $\mathrm{y}=80 \mathrm{~mm}$. Since the block has created between the region bounded by $\mathrm{y}=64 \mathrm{~mm}$ and $\mathrm{y}=74 \mathrm{~mm}$ so the pressure taken at $\mathrm{y}=80 \mathrm{~mm}$ fallsdramatically due to the presence of block in the main portal vein. This may cause of more shrinkage of the vein which stops blood circulation gradually. Its gives the most favorable picture of portal hypertension. 
The displayed fig.5 illustrates the change of velocity profiles for the partial blocked portal vein case which are taken from the mentioned regions. The velocity taken at $y=20 \mathrm{~mm}$ is in flatten shape with low magnitude and the velocity profile at $\mathrm{y}=80 \mathrm{~mm}$ is in distorted shape with high magnitude. Moreover the sharply increment of the velocity profile taken at $y=80 \mathrm{~mm}$ is a consequence of stuffing flow through the narrow flow path and the fluctuation of the velocity profile is an indication of back flow through the flow path. This back flow may introduce high blood pressure near the inlet region and may responsible for diminish of blood circulation.

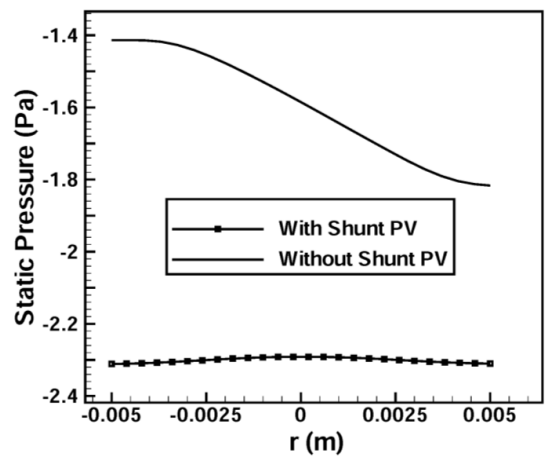

Fig.6: Comparison of pressure distributions for the two cases

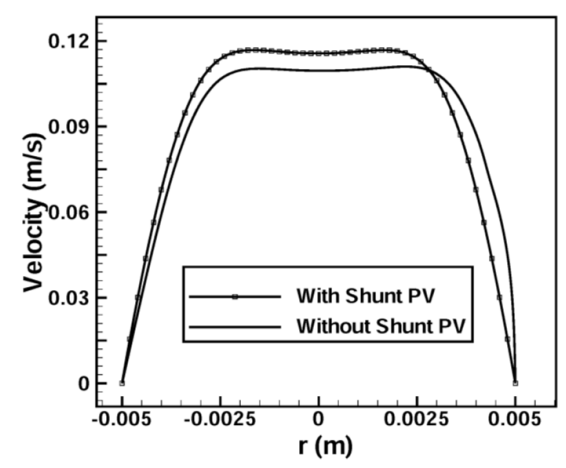

Fig.7: Comparison of velocity profiles for the two cases

Fig.6 and fig.7 show the assimilation of static pressure distribution and velocity profile for the blocked portal vein (or without-shunt PV) as well as with-shunt portal vein cases respectively. Fig.6 depicts the scenario of low pressure distribution for the with-shunt case compared to the withoutshunt portal vein case which are taken at $y=20 \mathrm{~mm}$, before the block region. After the creation of the shunt or bypass, there is a reduction of pressure before the blocked portal vein region.

Onward velocity profile is observed from the with-shunt portal vein case, in fig.7, compared to the without-shunt portal vein case. The with-shunt PV case provides the high velocity magnitude thereby low pressure, before the blocked region, compared to the without-shunt PV case.

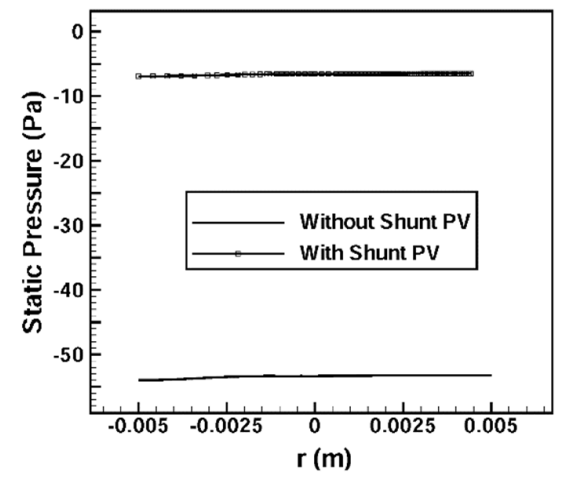

Fig.8: Comparison of pressure distribution after the block region for the two cases

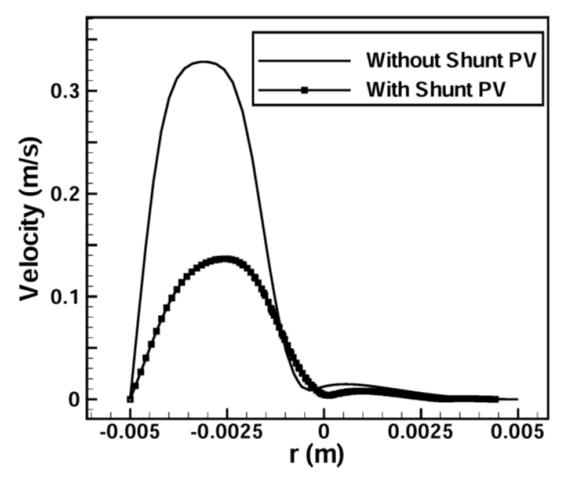

Fig.9: Comparison of velocity profile after the block region for the two cases 
Fig. 8 shows the assimilation of static pressure distributionsafter the thomobosed region together with fig.9 represents the comparison of velocity profiles for the two cases respectively. There is a high velocity beside the thomobosed region in the partial blocked portal vein (without shunt) but there is a comparatively low velocity (fig.9) for the with-shunt portal vein case. Flow variations of flow characteristics are produced by the creation of the bypass (or shunt).

In the vein, velocity as a function of distance along the vessel axis so velocity increases as the flow path become narrows for the without-shunt portal vein.After the creation of the bypass, the portal flow is followed by progressive diversion to the liver and subsequently reduce the velocity beside the thomobosed region.

It is observed that portal pressure is low, after the blocked region, for the without-shunt portal vein case.High velocity magnitude at thatregion has been responsible for this kind of pressure distributions. After the creation of the bypass, there is an increased of pressure for the with-shunt portal vein case. This increased of pressurewill give protection against shrinkage of the vein and might help to restore portal circulation through the main portal vein.

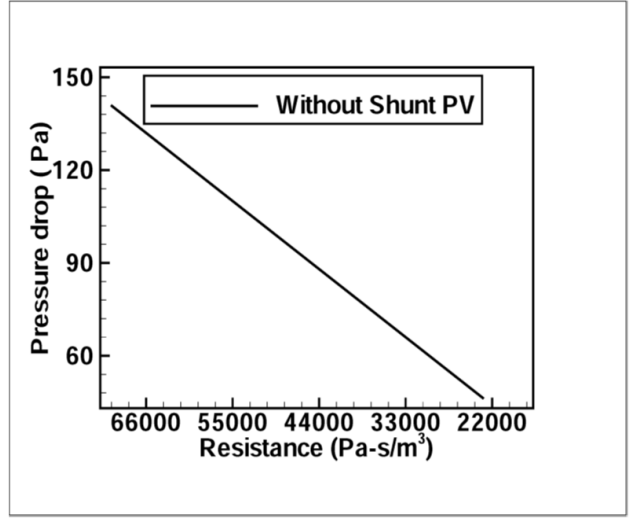

Fig.10: Resistance of flow for the without-shunt portal vein $(\mathrm{PV})$

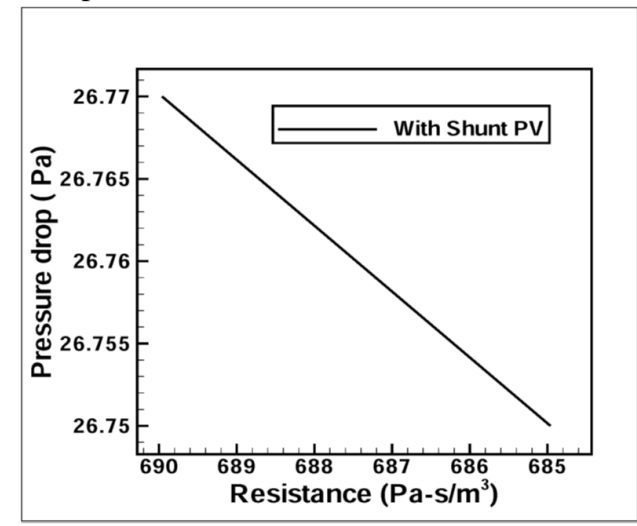

Fig.11: Resistance of flow for the with-shunt portal vein $(\mathrm{PV})$

Fig.10 and Fig.11 show the resistance of flow for without-shunt and with-shunt portal vein cases. Resistance controls the blood flow rate of the blood vessels. Resistance of blood vessel calculates here by using ohm's law. This law can be stated as - the higher the resistance, the slower the flow rate. The resistance of flow in case of without-shunt portal vein is higher due to the larger amount of reduction of cross-sectional area. In small width of the vessels, it is hard to push a lot of blood through it. It impliesincreased the pressure difference and decreased flow rate through the vessel.On the other hand, the resistance of flow in the case of with-shunt portal vein is very minimal. The creation of shunting is significantly worked to reduce the resistance of flow and hence the low resistance might decrease the portal venous pressure.

Under the normal circumstances, blood flow through the portal vein is split between the right branch of the portal vein (RPV) and left branch of the portal vein (LPV) in such way that the 
conservation of mass flow through the liver is maintained. Since the right lobe of the liver is twice than left lobe of the liver so the RPV carries much blood than LPV.

Table 4: The RPV and LPV Flow rate

\begin{tabular}{|l|c|c|}
\hline \multirow{2}{*}{} & \multicolumn{2}{|c|}{ Flow rate $\left(\mathrm{m}^{3} / \mathrm{s}\right)$} \\
\cline { 2 - 3 } & RPV & LPV \\
\hline Without-shunt portal vein & 0.60817668 & -0.39157073 \\
\hline With-shunt portal vein & 0.67945963 & 0.39090082 \\
\hline
\end{tabular}

Table 4 gives the flow rate of RPV and LPV for the without -shunt portal vein case as well as with-shunt portal vein case. It is noted that, the flow rate through the RPV and LPV for the without -shunt portal vein and with-shunt portal vein cases is almost constant. The constancy is due to the maintenance of conservation of mass through the portal vein. But the flow rate in LPV contains a minus sign for the without -shunt portal vein case. This minus sign indicates a decrease of pressure in the direction of flow through the LPV. The decreases of blood pressure through LPV may cause atrophy of the left lobe of liver. But for the with-shunt portal vein case, the flow rate indicates the restoration of blood flow through the LPV which may reduce the atrophy complex to the liver.

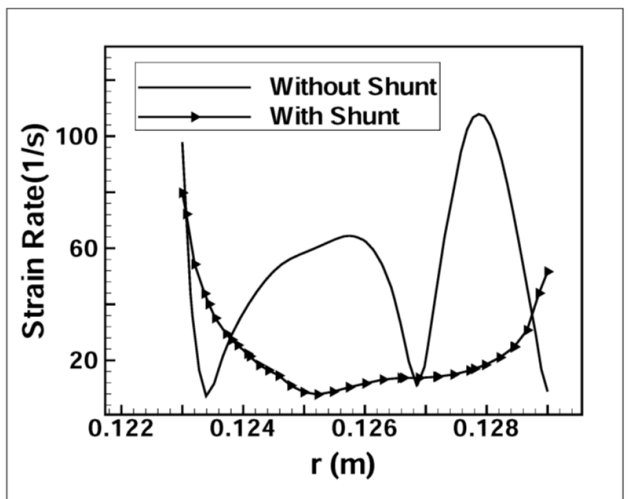

Fig.12: Comparisons Strain Rate for RPV

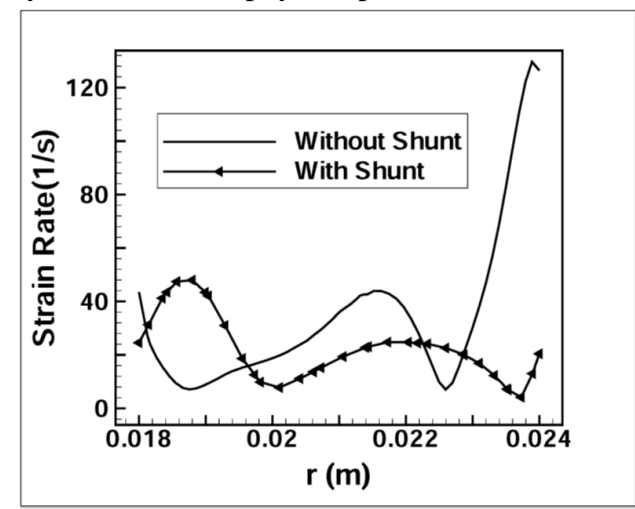

Fig.13: Comparisons Strain Rate for LPV

The variations of strain rate in the flow path for the right and left branches of the portal vein for the two cases has been depicted by fig.12 and fig.13. Since in the present model shear- thinning behavior of blood viscosity is assumed so the strain rate might be low in the flow. But for the without-shunt portal vein case, as shown in fig.12 and fig.13, the strain rate shows chaotic behavior with high magnitude along the mean flow path. At high shear rates, whole blood achieves its minimum viscosity value. In this situation, the RBCs (Red blood cell) maximally deform and align themselves to produce the maximum resistance to flow which may contribute to change of viscoelasticity property of blood.

After shunting (or with shunt) the strain rate shows a distinct behavior with low strain rate magnitude, shown in fig. 12 and fig. 13 , which may contribute to level the restoration of the original form of the RBC. 


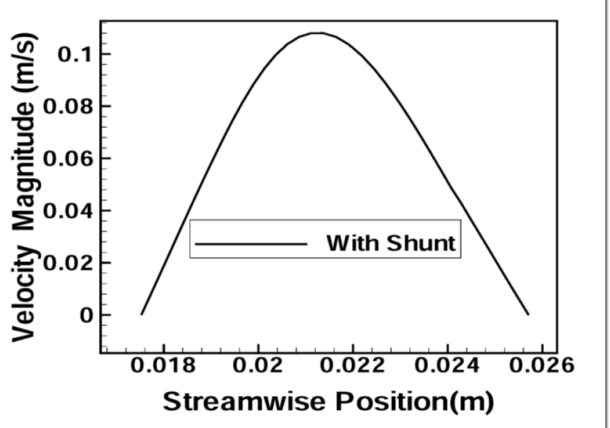

Fig.14: Velocity distribution in the shunt section

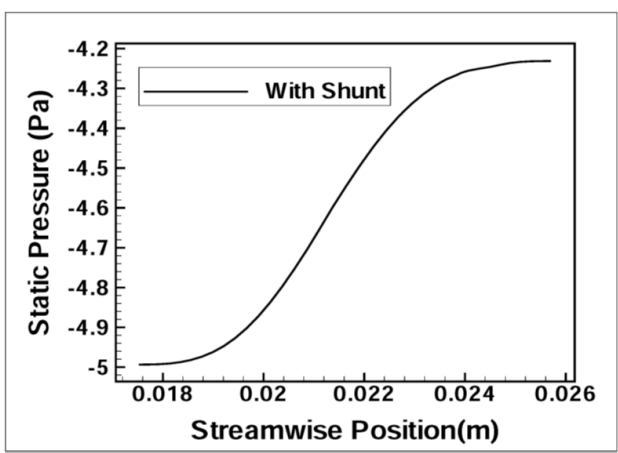

Fig.15: Pressure distribution in the shunt section

Fig.14 and Fig.15 revel the velocity distribution and the pressure distribution along the shunt section respectively. It is shown that the velocity profile is in parabolic shape and also the pressure profile is in non-linear form. From the pressure-flow relationship of the flow it can be said that there act less pressure gradient during the flow path which may lead smooth blood flow through the vein.

\section{Discussion}

All methods currently used or under development to deal with portal hypertension or its complications have their advantages and disadvantages, and they are more or less effective than other methods. It will be beneficial and logical for all treatment methods to be available in any medical center undertaking hepatic surgery, so that in each individual case the appropriate approach can be chosen. In some cases a combination of different treatments might be the correct approach, and the search for novel methods needs to continue. The aim of this study is to investigate ways to deal with the diminished blood pressure and look into possible ways to either improve the flow or to predict the impact of the condition on the outcome for the patient.

EHPVOcan be responsible for enlargement of spleen andvaricose veins in the stomach with severe bleeding from gastrointestinal varices, ascites, thrombocytopenia from hypersplenism and other coagulation disorders. Since the thrombosis is often limited to the portal trunk and lead to the shrink of vain so it will be a good practice to emphasize the importance of repairing the venous obstruction. After bypassing the blocked region a satisfactory flow through the left branches of the portal vein is observed. This will lead to an impressively rapid and progressive increase in the size of the intrahepatic left portal vein.

The use of main portal trunk for carrying blood due to the construction of shunt will be a great benefit of this shunting technique. This shunting (or bypassing) technique may function well in both preventing recurrence of upper gastrointestinal bleeding and in decompressing the enlarged spleen with resolution of the portal hypertension compare to the traditional shunting procedures. Wherever the thrombosis are formed in the main portal vein, this shunting scheme is potentially 
applicable. The shunt procedure signifies a further refinement in surgical approach to theproblem of portal hypertension with no underlying significant liver disease.

\section{Conclusions}

It has been suggested from studies that EHPVO may have sublet deficiencies in liver function and that chronic diversion of portal blood away from the liver may lead to progressive liver dysfunction. This study shows that direct bypassing of portal vein thrombosis is possible treatment of the complications of portal hypertension with EHPVO. Restoration of the portal flow to the liver and normalization of the portal venous pressure is a majoradvantage of this bypassing (or shunting) procedure. The shunt may provide immediate palliation from massive medically untreatable ascites as well as from the variceal bleeding, which had been poorly controlled through conventional surgical therapy. Clinical studies are highly recommended for assessing the practical usefulness of the model.

\section{REFERENCES}

[1] Balfour G.W. and Stewart T.G., "Cases of enlarged spleen complicated with ascites, both depending upon varicose dilatation and thrombosis of the portal vein”, Edinb Med J, 14(1869), 589-598.

[2] Becker, Y \& Reed, G, "The role of elective operation in the treatment of portal hypertension", American Surgeon, 62(3) (1996), 313-48.

[3] Belli L, Puttini M, Mami A. "Extrahepatic portal obstruction. Clinical experience and surgical treatment in 105 patients.” J Cardiovasc Surg, 21(1980), 439-448.

[4] C.C. Botara, T. Vasileb, S. Strangeub, S. Clichicic, P. S. Agachia, R. Badeab, P. Mircead and M. V.Cristeaa, "Validation of CFD simulation results in case of portal vein blood flow", 20" European symposium on computer Aided process Engineering ESCAPE 20(2010).

[5] D. Wang and J. Bernsdorf."Lattice Boltzmann simulation of steady Non-Newtonian blood flow in a 3D genericstenosis case”. Computers and Mathematics with Applications, 58(2009), 1030-1034.

[6] Dilawari JB, Chawla YK., "Spontaneous (natural) splenoadrenal shunts in extrahepatic portal venous obstruction: A series of 20 cases", Gut,28(1987), 1198-1200.

[7] Evans S, Stovroff M, Heiss K, et al, "Selective distal splenorenal shunts for intractable variceal bleeding in pediatric portal hypertension." JPediatrSurg, 30(1995), 1115-1118.

[8] Giorgio Ricci, Flavia Pigo, Angelo Rossi, Gianluca Bersani, Vittorio Alvisi,, "Non-cirrhotic extrahepatic portal vein thrombosis: a 6-year long case history", Int J Hematol, 91(2010), 338-339.

[9] J. de Ville de Goyet, D. Alberti, D. Falchetti, W. Rigamonti, L. Matricardi, P. Clapuyt, E. M. Sokal,J. B. Otte and G. Caccia, "Treatment of Extrahepatic Portal Hypertension in Children by Mesenteric-toleft Portal Vein Bypass: a New Physiological Procedure”, Eur J Surg, 165(1999), 777-781.

[10] J. Vimmr and A. Jonásová, "Non-Newtonian effects of blood flow in complete coronary and femoral bypasses". Mathematics and Computers in Simulation, 80 (2010), 1324-1336.

[11] Mst. Khorseda Aktar and Md. Tajul Islam,“A computational analysis of blood flow through portal vein under normal and extrahepatic obstructions”, J. Bangladesh Acad. Sci., 41(2)(2017), 183-199.

[12] Ohnuma N, Takahashi H, Tanabe M. et al,"Endoscopic variceal ligation using a clipping apparatus in children with portal hypertension”, Endoscopy,29(1997), 86-90.

[13] Orloff MJ, Orloff MS, Girard B, Orloff SL., "Bleeding esophagogastric varices from extrahepatic portal hypertension: 40 years' experience with portal-systemic shunt”. J Am Coll Surg, 194(2002), 717-728. 
[14] Petkova, A. Hossain, J. Naser, and E. Palombo, "CFD modeling of blood flow in portal vein hypertension with and without thrombosis". Third International Conference on CFD in the minerals and Process Industries, 527-530(2003).

[15] Poddar U., Thapa B.R., Rao K.L. and Singh K., "Etiological spectrum of esophageal varices due to portal hypertension in Indian children: is it different from the West", $J$ Gastroenterol Hepatol, 23(2008), 1354-1357.

[16] Sherlock, S, "Portal circulation and portal hypertension", GUT, 19(1978), 70-83.

[17] Shilyansky J, Roberts E, Superina RA, "Distal splenorenal shunts for the treatment of severe thrombocytopenia from portal hypertension in children." J Gastrointest Surg,3(1999), 167-172.

[18] Superina RA, Weber JL, Shandling B, "A modified Sugiura operation for bleeding varices in children.” J Pediatr Surg, 18(1983), 794-799.

[19] Thuluvath, P J, Bal, J S, Mitchell, S, Lund, G \& Venbrux, A, "TIPS for management of refractory ascites: response and survival are both unpredictable", Dig Dis Sci, 48(3)(2003), 542-450.

[20] Uchiyama M, Iwafuchi M, Ohsawa $Y$, et al, "Long-term results after nonshunt operations for esophageal varices in children." J Pediatr Surg, 29(1994), 1429-1433.

[21] Yachha SK, Sharma BC, Kumar M, et al, "Endoscopic sclerotherapy for esophageal varices in children with extrahepatic portal venous obstruction: a follow-up study". J Pediatr Gastroenterol Nutr, 24(1997), 49-52. 